\title{
Cue-based preparation and stimulus-based priming of tasks in task switching
}

\author{
IRING KOCH \\ Max Planck Institute for Human Cognitive and Brain Sciences, Munich, Germany \\ and \\ ALAN ALLPORT \\ University of Oxford, Oxford, England
}

\begin{abstract}
In this study, we investigated the interaction of three different sources of task activation in precued task switching. We distinguished (1) intentional, cue-based task activation from two other, involuntary sources of activation: (2) persisting activation from the preceding task and (3) stimulus-based task activation elicited by the task stimulus itself. We assumed that cue-based task activation increases as a function of cue-stimulus interval (CSI) and that task activation from the preceding trial decays as a function of response-stimulus interval. Stimulus-based task activation is thought to be due to involuntary retrieval of stimulus-associated tasks. We manipulated stimulus-based task activation by mapping each of the stimuli consistently to only one or the other of the two tasks. After practice, we reversed this mapping in order to test the effects of item-specific stimulus-task association. The mapping reversal resulted in increased reaction times and increased task shift costs. These stimulusbased priming effects were markedly reduced with a long CSI, relative to a short CSI, suggesting that stimulus-based priming shows up in performance principally when competition between tasks is high and that cue-based task activation reduces task competition. In contrast, lengthening the response-cue interval (decay time) reduced shift costs but did not reduce the stimulus-based priming effect. The data are consistent with separable stimulus-related and response-related components of task activation. Further theoretical implications of these findings are discussed.
\end{abstract}

Action control is shaped by numerous interacting factors. These are sometimes partitioned into internal factors (e.g., current intentions, goals) and external factors, such as the tendency of situations (or stimuli) to prime actions associated with them. Recently, the interaction of these factors has been studied using the task-switching paradigm. In task switching, performance in repeated tasks is compared with performance when there is a requirement to switch between different tasks. Typically, reaction time (RT) and error rate are greater on task switch than on repetition trials. These shift costs appear in many studies, in which a variety of tasks and paradigms have been used (see, e.g., Monsell, 2003, for a review).

\section{Interactive Processes in Task Switching}

It has been suggested that shift costs in task switching are related to a process of reconfiguring the task set (see, e.g., Goschke, 2000; Meiran, 1996; Rogers \& Monsell, 1995). The main empirical support for this suggestion has come from studies exploring the effects of the

\footnotetext{
The authors thank Erik Altmann, Guido Band, and an anonymous reviewer for helpful comments, as well as Stefanie Schuch and Andrea Philipp for discussing this work. Correspondence concerning this article should be addressed to I. Koch, who is now at the Institute of Psychology, RWTH Aachen University, Jägerstrasse 17-19, D-52056 Aachen, Germany (e-mail: koch@psych.rwth-aachen.de).
}

time available for task preparation. For instance, Rogers and Monsell varied the temporal interval from the preceding response to the onset of the next task stimulus (response-stimulus interval, RSI) in predictable, instructed task sequences (e.g., AABBAABB etc.) and found that longer RSIs were associated with reduced shift costs. They took this reduction of shift costs with long RSIs as evidence for advance preparation for the upcoming task.

However, lengthening the RSI provides time not only for active preparation of the upcoming task, but also for changes relating to the preceding task, such as the decay of activation relevant to that task (Meiran, 1996). Both of these processes could, in principle, affect the size of shift costs. To dissociate the influence of preparation and decay, Meiran (1996) used explicit instructional cues in a random task sequence.

Decay of task activation. The cuing paradigm allows the potential effects of decay time on shift costs to be examined, independently of active preparation. To do so, the cue-to-stimulus interval (CSI), which represents the time available for cue encoding and task preparation, is held constant while the prior response-to-cue interval (RCI) is varied. In fact, it has been found that shift costs decrease with increasing RCI (see, e.g., Koch, 2001; Meiran, Chorev, \& Sapir, 2000), in accord with the idea that task activation decays over time following execution of a response. The assumption is that persisting task activation 
from the preceding trial dissipates more with a long than with a short RCI, so that when a new task set needs to be activated on a task switch trial, there is reduced competition from the preceding task after a longer RCI, resulting in reduced shift costs.

Cue-based preparation of tasks. The cuing paradigm has also been used to demonstrate the effects of preparation of the upcoming task, independently of decay time effects. To do this, the CSI is varied while the RSI is held constant (i.e., by varying RCI inversely to CSI). Studies in which this procedure has been used have shown that shift costs decreased with increasing CSI (e.g., Koch, 2001; Meiran, 1996; Meiran et al., 2000), in accord with the view that preparation of task set, prior to stimulus onset, is indeed an important factor affecting shift costs (Rogers \& Monsell, 1995). Clearly, cue encoding is a constituent part of this CSI-dependent process. Recent studies in which more than one cue has been used per task in the cued task-switching paradigm have suggested that part of the reduction in RT with increasing CSI may be attributed to the effects of cue encoding (but see Logan \& Bundesen, 2003, 2004; Mayr \& Kliegl, 2003).

Both of these processes, cue encoding and cue-based task preparation, are often referred to as voluntary or intentional, on the grounds that the verbal instructions to the participant (before the experiment) explicitly emphasize the association between the cue and the task that it signals. Such associations typically remain consistent, however, so that cue-based preparation in the task-cuing paradigm can also be viewed as an associative process by which the cue activates (or retrieves) the task associated with it (see, e.g., Mayr \& Kliegl, 2003). The intentional status of this cuebased activation, we suggest, is reflected in the fact that, once activated, the cue-elicited bias for the correct task is sustained, rather than cue-task retrieval being performed by a special (nonassociative) mechanism.

Stimulus-based priming of tasks. Cue-based task activation can be distinguished from involuntary or nonintentional activation of tasks, elicited by the task stimuli themselves. The assumption is that individual task stimuli become implicitly associated with the tasks and task contexts in which they have previously occurred. Where this association between stimuli and tasks is unique, the stimuli may thus acquire a task activation function similar to, and in addition to, the cue-based activation of the correct task (see, e.g., Allport \& Wylie, 2000; Rogers \& Monsell, 1995; Waszak, Hommel, \& Allport, 2003). We will refer to the behavioral effects of such stimulus-task associations as stimulus-based priming.

Of course, a given stimulus may have been associated previously with a different task, other than the currently "correct" one; in this case, stimulus-based priming is liable to interfere with, rather than to facilitate, activation of the correct task. Indeed, in studies of task switching, the same stimuli typically occur in both tasks, so that stimulus-totask associations are liable to be acquired in respect of both tasks. For example, in Stroop color-word tasks, the same compound color-word stimuli typically occur in both the word-reading and the color-naming tasks (e.g., Allport, Styles, \& Hsieh, 1994). The stimulus is thus liable to reactivate both the correct task and the competing task, so that it is potentially an important source of interference (see also the concept of exogenous task cuing, suggested by Rogers \& Monsell, 1995).

Recently, Allport and Wylie (2000) and Waszak et al. (2003) have shown that such stimulus-based priming of a competing task can, indeed, contribute substantially to interference in task switching. Allport and Wylie (2000) used compound color-word Stroop stimuli and required their participants to switch between color naming and word reading (see also Allport et al., 1994). The compound (Stroop) stimuli were always incongruent (such as the word GREEN presented in blue color), which means that they were associated with different responses in these two tasks, whereas congruent stimuli (e.g., GREEN presented in green color) would be associated with one and the same response for both tasks. In Allport and Wylie's (2000) Experiment 5, some color-words were uniquely associated with the word-reading task, whereas the remaining ( primed) color-words had also occurred, as distractors, in the color-naming task. Allport and Wylie (2000) found that word-reading RT in response to color-words primed by their occurrence in the competing task was significantly greater, on task switch trials, than that to colorwords that had not appeared in the context of the other task (unprimed stimuli). Similar effects in word reading have been found by Waszak et al. (2003), using picture-word Stroop stimuli instead of color-word Stroop stimuli. They found stimulus-based priming on switch trials even after a single prior exposure to a stimulus in the context of the competing task and with intervals of more than 200 trials between prime and probe events. These authors observed stimulus-based priming effects also for congruent stimuli, suggesting that this priming is not confined to S-R associations but extends to the task level, too. Further studies by these authors have confirmed and extended their earlier findings (Waszak, Hommel, \& Allport, 2004, 2005).

The aim of the present study was to introduce a novel manipulation of stimulus-based priming in the context of task switching, using the cuing paradigm. By means of this manipulation, we were able to examine the interaction of stimulus-based priming (i.e., involuntary task activation) with two other, hitherto more widely studied sources of task activation: (1) cue-based task activation (over increasing CSI) and (2) decay of task activation from the preceding trial (over increasing RCI). In the following sections, we first will describe our novel method for manipulating stimulus-task associations; we then will lay out the theoretical framework that provided the motivation for this study, its experimental rationale, and finally, a set of predictions based on our theoretical approach.

\section{A Novel Paradigm for Manipulating Stimulus- Task Associations}

In our study, we used a pair of numerical judgment tasks that were approximately symmetrical in task diffi- 
culty, unlike the Stroop tasks used in the earlier studies. The participants judged whether a given digit from 1 to 9 (excluding 5) belonged to the category greater than 5 or less than 5 (magnitude task) or whether it belonged to the category odd or even (parity task). They signaled their judgments by pressing one of two response keys (the same pair of keys for both tasks). Depending on the instructed mapping of category to response ( $\mathrm{C}-\mathrm{R}$ mapping), the two judgments (in Tasks A and B, respectively) pertaining to a given digit could thus be assigned to the same response key (congruent responses) or to different keys (incongruent responses).

We manipulated stimulus-based priming by assigning each stimulus uniquely to one of the two tasks (consistent stimulus-task mapping; see, e.g., Shiffrin \& Schneider, 1977, for related manipulations in a different context). For example, the digit 3 is both odd and lower than 5 . For a given participant, however, this digit would appear, say, exclusively in the context of the parity task and never in the magnitude task. Through associative learning, the digit 3 should thus come to activate the parity task and the odd category. After several blocks of practice, however, all these consistent stimulus-to-task mappings were abruptly reversed, so that, after the mapping reversal, the digit 3 in our example appeared, from then on, only in the context of the magnitude task. Hence, the previous (positive) stimulus-task priming should now become negative, priming the competing, incorrect task. This stimulus-to-task mapping reversal, changing positive to negative stimulusbased priming, should produce a negative transfer effect, thus providing our measure of stimulus-based priming. Importantly, for stimuli with congruent (i.e., identical) responses in the two tasks, the mapping reversal does not change the associated overt response but only the associated judgment task. Any effects of mapping reversal for response-congruent stimuli would thus indicate taskspecific priming.

Note that the earlier studies on stimulus-based priming in task switching (i.e., Allport \& Wylie, 2000; Waszak et al., 2003) focused on testing these priming effects on the highly overlearned task of word reading; they were not designed to symmetrically examine the corresponding effects on the less well practiced tasks of color naming (Allport \& Wylie, 2000) or picture naming (Waszak et al., 2003). That is, the evidence to date is restricted mainly to priming effects on the stronger (or more dominant) task within pairs of tasks that differed very substantially in strength (i.e., Stroop tasks). In contrast, in our study, the two tasks were relatively symmetrical in strength, and more important, the experimental manipulation of stimulus-task associations was fully balanced with respect to the two tasks.

\section{Theoretical Framework and Rationale}

Involuntary, stimulus-based priming is one potential source of task activation in task switching. However, its effects (like those of carryover priming from the preceding trial) are seen largely in RTs, rather than in terms of which task is ultimately executed. In most cases, the actual task performed is determined by the explicit task cue; in contrast, what determines RT is the time needed for the system to settle to an internally consistent state: the greater the competition, the longer the RT (Allport \& Wylie, 1999; Gilbert \& Shallice, 2002; Ward, 1999).

The critical mechanism by which the task cue exerts its determining influence, we suggest, is via its sustained activation of the associated task; involuntary, stimulusbased activations, by contrast, are relatively transient. This idea-that intentional processes differ from unintentional processes in the brain by virtue of their sustained character-is certainly not new. Van der Heijden (1981) was an early exponent of this view, with respect to visual attention. More recently, this idea has received increasing theoretical and empirical support (Braver \& Cohen, 2000; Braver, Reynolds, \& Donaldson, 2003; Duncan, Humphreys, \& Ward, 1997; Miller \& Cohen, 2001; Ward, 1999).

Cue-based processes activate the correct task by virtue of the cue's unique cue-task association. This retrieval function of the cue is quickly learned during the experiment, so that the task cue can reliably exert a bias on the competition among tasks (Meiran, 2000a; Miller \& Cohen, 2001), and this bias is then sustained, at least until response execution. However, we also assume that task activation is dynamic, in that cue-based task activation increases as a function of CSI, and that this activation also declines after successful task performance (i.e., after response execution). On a task switch, there is residual activation of the preceding (now incorrect) task, so that at a short RSI the level of task competition is high; with increases in the RSI, however, decay of the preceding task activation will gradually reduce this source of task competition. Thus, both cue-based preparation (with an increasing CSI) and decay of the preceding task (with an increasing RSI) should act to reduce conflict-hence, to reduce RT on task switch trials, albeit for different reasons. A long CSI increases the activation of the correct task, whereas a long RSI decreases the activation level of the incorrect task (see Koch, 2001; Meiran et al., 2000).

On the basis of this theoretical framework, our objective was to study how these two dynamic activation processes - the buildup of cue-based task activation and passive decay of activation from the preceding trialinteract with stimulus-based priming. As was described above, stimulus-based priming has already been demonstrated to produce substantial interference effects in task switching (e.g., Waszak et al., 2003). However, previous studies (Allport \& Wylie, 2000, Experiment 5; Waszak et al., 2003, 2004, 2005) used fixed (and relatively long) task preparation intervals and also fixed decay intervals.

The initial motivation for such a study was straightforward. We reasoned that, insofar as stimulus-based priming operates at the same functional level (or levels) as do these other two sources of time-varying task activation, all three processes might be expected to show a systematic (time-varying) pattern of interactions. The resulting data set should thus provide a powerful set of constraints for any theory of task control. 
In the present study, we explored the interactions among these three different sources of task activation, as a function of varying CSI and RSI, by comparing the performance of three groups of participants. The manipulation of stimulusbased priming was the same in all three groups. The groups differed in the duration of the CSI (cue-based preparation), with the overall RSI held constant, or in the duration of the overall RSI (allowing the task activation from the preceding trial to decay), with cue-based preparation time held constant. Thus, in one between-group comparison, the two groups differed only in the duration of the CSI (cue-based task preparation interval), either short $(100 \mathrm{msec})$ or long (900 msec), with total RSI held constant at 1,000 msec. These two groups are referred to as the 900-100 and 100900 groups, respectively, where the first number denotes the RCI and the second number denotes the CSI. Comparison between these two groups thus permitted us to observe stimulus-based priming when cue-based task activation was still weak (100 msec after the appearance of the task cue) and when it could be assumed to be relatively strong (900 msec after the appearance of the task cue), respectively. In our second between-group comparison (the 900-100 and 100-100 groups), CSI was held constant at $100 \mathrm{msec}$, but the overall RSI (decay time) varied (1,000 vs. $200 \mathrm{msec}$ ). Comparing performance of these two groups allowed us to observe the influence of the preceding task and its decay on stimulus-based priming and shift costs.

We made two sets of predictions on the basis of the theoretical assumptions outlined above. The first set of predictions was about the expected interactions between stimulus-based priming, response congruence, and task switching; the second set of predictions was about the possible interactions between stimulus-based priming and CSI and RSI, respectively.

First, as was discussed above, we assumed that stimulusbased priming operates at the level of tasks (rather than responses). Accordingly, we predicted (1) that stimulusbased priming would affect response-congruent, as well as incongruent, trials. We also predicted (2) that the reversal of stimulus-task mappings would have a stronger effect on task switch trials-when task competition was already strong - than on task repetitions: in other words, that stimulus-based priming would increase switch costs.

Our second set of predictions concerned possible interactions between our manipulation of stimulus-based priming and the (between-group) variation of CSI and RSI. We assumed that an increasing CSI permits an increasing bias in favor of the correct task and against the competing task, thus reducing the degree of between-task competition (see also Gilbert \& Shallice, 2002). That is, cue-based task activation can reduce (although perhaps not eliminate) task competition. We therefore predicted (3) that, after a long CSI, stimulus-based priming of the competing task should have relatively little effect against this already strongly imposed bias. In contrast, at a short CSI, when the task cue has not yet had time to bias the task competition strongly, stimulus-based activation of the incorrect task should increase the level of between-task conflict, thus substantially increasing RT. Moreover, following the same reasoning that led us to Prediction 2 above, we also predicted (3a) that increasing the CSI should affect stimulus-based priming most strongly on switch trials.

Finally, as regards the possible interaction between our manipulation of stimulus-based priming and RSI (decay time), our basic assumption was that both stimulus-based priming and carryover priming from the preceding trial operate at the same level (i.e., by activating one or the other of the competing tasks). Thus, we expected that these two variables should interact. To anticipate the results, however, we found that these two variables had independent effects on performance. We will present a speculative account for this finding in the Discussion section.

In summary, the present study was designed to explore the main effects of and interactions among three major processes in task switching (intentional task preparation, task decay, and involuntary stimulus-based priming). Exploration of the interactions among these processes should provide important empirical constraints for theories of executive control.

\section{METHOD}

\section{Participants}

Forty-eight participants ( 34 of them female and 14 male; mean age, 23.7 years) took part and received $€ 5$ each. Sixteen participants were randomly assigned to each of the three groups: 100-900, 900100 , and 100-100.

\section{Stimuli and Tasks}

The stimuli were the digits $1-9$, excluding 5 . Their height was $0.8 \mathrm{~cm}$. They were centrally presented inside a square or diamond frame measuring $3.8 \times 3.8 \mathrm{~cm}$ on a 15 -in. computer screen connected to an IBM-compatible PC. Viewing distance was $60 \mathrm{~cm}$. The tasks were to decide (1) whether the digit was odd or even (parity) or (2) whether it was greater or less than 5 (magnitude). Responses were made by pressing either a left or a right key with the left or the right index finger, respectively. The response keys measured $1.7 \times$ $1.7 \mathrm{~cm}$, separated by $3.3 \mathrm{~cm}$.

\section{Procedure}

The participants were informed that the stimuli would be individually presented on the screen inside an outline frame, whose shape served as the instructional cue to the task. A diamond frame indicated the high-low task, and a square frame the odd-even task. The task cue appeared 100 or $900 \mathrm{msec}$ after the preceding response (RCI) and 100 or $900 \mathrm{msec}$ prior to stimulus onset (CSI), depending on the between-subjects experimental condition. The cue and stimulus remained on the screen until the response was made. Stimulus presentation order was random, with the constraint that repetition of the same digit on immediately successive trials was excluded. Task (magnitude vs. parity) also varied randomly from trial to trial.

The fixed assignment of the eight individual stimuli to the two tasks (i.e., four stimuli for each of the two tasks) was balanced over the four judgment categories (high, low, odd, and even), so that one stimulus was congruent and the other incongruent for each of the four categories. This assignment remained constant for each participant. Four different assignments were used across participants, counterbalanced over the four different stimulus-category-toresponse $(\mathrm{C}-\mathrm{R})$ mappings. The participants were provided with a reminder of the $\mathrm{C}-\mathrm{R}$ mappings (e.g., odd-left, even-right), placed below the screen. They were told that their responses should be fast but accurate. An error message appeared for $500 \mathrm{msec}$ at the bottom of the screen (thus delaying the next trial) if the participants committed an error. 
The participants performed 12 initial practice trials, followed by the experimental blocks of 96 trials each. After each block, the participants received RT feedback. After four training blocks, the stimulus-to-task mapping was reversed in Block 5. The experiment took about $40 \mathrm{~min} .^{1}$

\section{Design}

The independent within-subjects variables were task transition (switch vs. repeat), response congruence (congruent vs. incongruent), and stimulus-to-task mapping. To manipulate stimulus-to-task mapping (as was described in the introduction), we gave four blocks of training with one consistent stimulus-to-task mapping and then reversed the mapping in Block 5. The critical comparison was thus between Blocks 4 and 5 (mapping reversal). The between-subjects variable (group) had three levels: 100-900, 900-100, and 100-100, but we analyzed the data in two nonorthogonal contrasts: 100-900 versus $900-100$ (preparation contrast) and $900-100$ versus $100-100$ (decay contrast). ${ }^{2}$ The dependent variables were RT and errors. Significance was tested at $\alpha=.05$.

\section{RESULTS}

For data analysis, we discarded the first two trials of each block and trials immediately following an error, and for the RT analysis we also dropped trials associated with an error, as well as outliers $(\mathrm{RT}>3,000 \mathrm{msec}$; fewer than $1 \%)$.

In a first analysis, we examined the effect of task difficulty. Averaged across all variables, we found that performance in the magnitude task was somewhat faster than that in the parity task $[M=767 \mathrm{msec}, S E=29 \mathrm{msec}$, vs. $M=805 \mathrm{msec}, S E=29 \mathrm{msec} ; t(47)=2.63, p<.05]$ and less error prone $[M=4.0 \%, S E=0.05$, vs. $M=5.5 \%$, $S E=0.05 ; t(47)=3.52, p<.01]$. This finding suggests that, overall, the magnitude task was slightly easier to perform than the parity task. However, as compared with the very marked asymmetry in Stroop versus non-Stroop task pairings, we believe that this difference in task difficulty is relatively minor. More important, our manipulation of stimulus-based priming applied in exactly the same way to both tasks, whereas in previous studies (e.g., Waszak et al., 2003) the priming manipulation was designed so that the effects of between-task priming were to be observed primarily in the stronger task. Thus, because our experimental manipulation was completely balanced with respect to the two tasks, we collapsed the data across the task variable in the theoretically relevant analyses to be reported next.

Figure 1 shows RT as a function of block and task transition for each of the three groups. The purpose of Blocks 1-4 was to induce specific stimulus-task associations. Clearly, there were large and monotonic practice benefits over these four blocks (see Koch, 2001). However, because over Blocks 1-4 the effects of item-specific stimulus-task learning are not separable from those of more general task practice (including, e.g., learning the category response rules: odd $\rightarrow$ left, even $\rightarrow$ right, etc.), we will not report a statistical analysis for these data. The crucial comparison pertains to the effects of reversing the item-specific stimulus-task mappings between Blocks 4 and 5. This comparison separates the effects of itemspecific stimulus-task learning from all other effects of task practice, which should continue to be positive from Block 4 to Block 5, since all other features of these tasks remained unchanged. A performance cost between Blocks 4 and 5 can be due only to the reversal of the previously learned stimulus-based associations. Given that all other practice-related benefits should continue to be positive and, thus, counteract the expected negative effect of reversing stimulus-task mappings, it seems safe to assume that our procedure yields a conservative measure (an underestimate) for the size of the stimulus-based priming effect. To test this priming effect, we ran an ANOVA on RTs from Blocks 4 and 5, with task transition, mapping reversal, and response congruence as independent within-

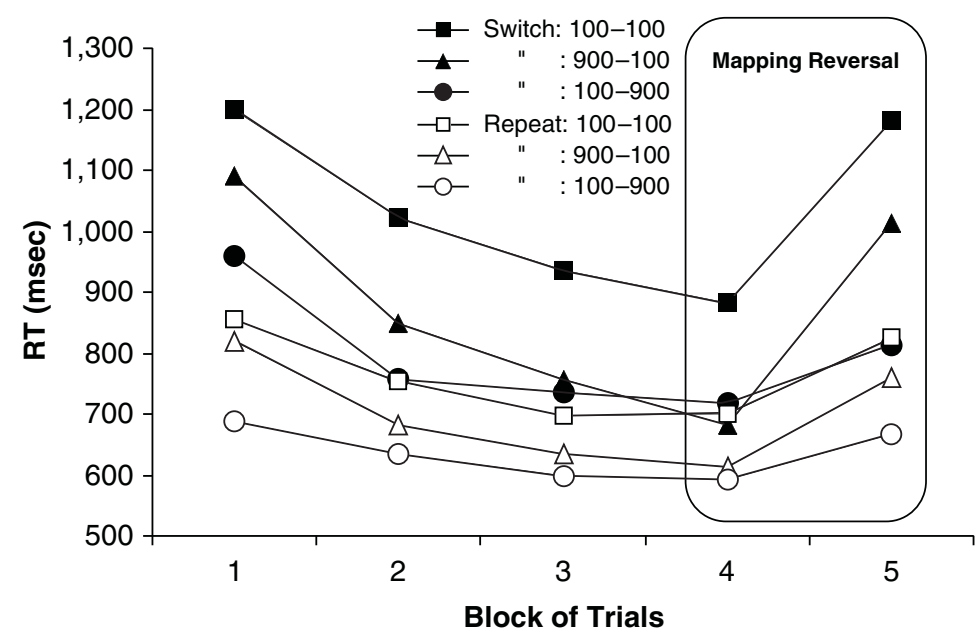

Figure 1. Mean reaction times (RTs) as a function of block of trials, task transition, and group. The comparison of performance in the 100-900 and 900-100 groups refers to the preparation contrast, whereas the comparison of performance in the $900-100$ and $100-100$ groups refers to the decay contrast. 
subjects variables and group as the between-subjects variable. The data are shown in Figure 2.

We will present the results in two major sections below, in which we will report the results of two nonorthogonal contrasts. First, we will focus on the interaction between stimulus-based priming and cue-based task preparation by comparing performance in the 100-900 and 900-100 groups. We then will analyze the interaction between stimulus-based priming and decay of activation of the preceding task by comparing performance in the 900-100 and 100-100 groups.

\section{Stimulus-Based Priming and Cue-Based Task Preparation}

We ran an ANOVA on Blocks 4 and 5, with RT as the dependent variable and with independent variables of mapping reversal (practiced vs. reversed, Blocks 4 vs. 5), task transition (task switch vs. repetition), response congruence, and group (900-100 vs. 100-900; see the left and middle panels of Figure 2).

The ANOVA revealed significant main effects of task transition $\left[F(1,30)=40.92, M S_{\mathrm{e}}=34,821\right]$ and response congruence $\left[F(1,30)=17.25, M S_{\mathrm{e}}=7,472\right]$. RT was longer in response-incongruent than in response-congruent trials $(M=755 \mathrm{msec}, S E=29 \mathrm{msec}$, vs. $M=710 \mathrm{msec}$, $S E=31 \mathrm{msec})$.

The ANOVA also revealed a main effect of mapping reversal $\left[F(1,30)=78.70, M S_{\mathrm{e}}=21,264\right]$, with longer

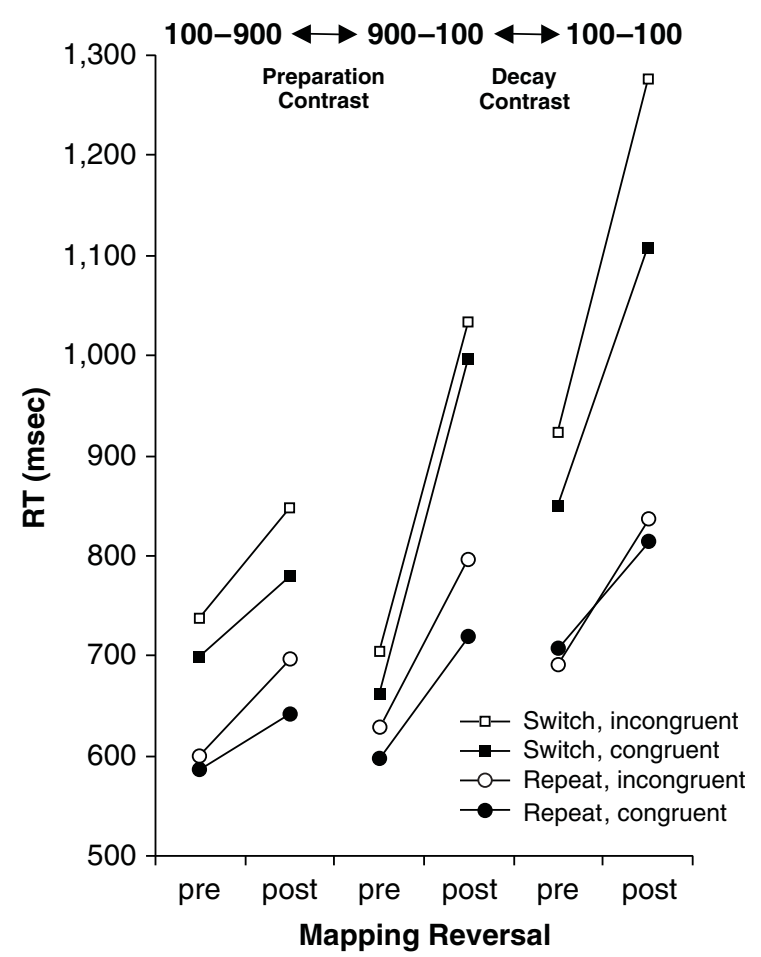

Figure 2. Mean reaction times (RTs) as a function of mapping reversal, task transition, response congruence, and group. The label "pre" refers to the prereversal Block 4, whereas "post" refers to the postreversal Block 5.
RTs after the mapping reversal than before the reversal $(M=814 \mathrm{msec}, S E=28 \mathrm{msec}$, vs. $M=652 \mathrm{msec}, S E=$ $34 \mathrm{msec})$. This effect did not significantly interact with response congruence $\left[F(1,30)=2.75, M S_{\mathrm{e}}=4,374, p>.1\right]$. The stimulus-based priming effect was numerically slightly larger for the response-incongruent stimuli, but when we tested the priming effect separately for the responsecongruent stimuli alone, the pattern of effects was the same as that in the complete analysis. This finding - that the effect of mapping reversal was statistically as large for responsecongruent as for response-incongruent stimuli-supports our assumption (Prediction 1) that stimulus-based priming affects the task level and cannot be due merely to stimulusbased priming of the previously associated responses, or direct S-R mappings (see also Waszak et al., 2003).

Mapping reversal of individual stimuli to tasks interacted with task transition $\left[F(1,30)=9.76, M S_{\mathrm{e}}=\right.$ $17,517]$, so that shift costs increased twofold from Block 4 to Block 5 (from 98 to $201 \mathrm{msec}$ ), thus clearly supporting Prediction 2.

For the present purpose, the effect of cue-based preparation time - that is, the between-group comparison-is crucial. There was no significant main effect of group $(p>.25)$, but group interacted with mapping reversal $\left[F(1,30)=17.49, M S_{\mathrm{e}}=21,264\right]:$ The increase in RT following mapping reversal (i.e., the stimulus-based priming effect) was much larger for the 900-100 group than for the 100-900 group. That is, after a short preparation interval (the 900-100 group) the stimulus-based priming effect was $238 \mathrm{msec}(M=886 \mathrm{msec}, S E=32 \mathrm{msec}$, vs. $M=$ $648 \mathrm{msec}, S E=37 \mathrm{msec}$ ), as compared with only $85 \mathrm{msec}$ $(M=741 \mathrm{msec}, S E=46 \mathrm{msec}$, vs. $M=656 \mathrm{msec}, S E=$ $57 \mathrm{msec}$ ) after a longer preparation time (group 100-900). This finding suggests that cue-based preparation biases the task competition in favor of the upcoming task, in advance of the stimulus, so that, after a long CSI, any stimulusbased priming of the incorrect task occurs in a situation in which the correct task is already dominant.

However, as Figure 2 (left and center graphs) makes clear, this contrast was very much more marked for task switch trials than for task repetition trials. Cue-based preparation time thus strongly modulated the impact of stimulus-mapping reversal on shift costs (Prediction 3a); that is, there was a three-way interaction of group, mapping reversal, and task transition $\left[F(1,30)=6.35, M S_{\mathrm{e}}=\right.$ 17,517]. As Figure 2 shows, the effect of mapping reversal on shift costs was reduced by the long preparation interval in the 100-900 group, as compared with the 900-100 group. (Mapping reversal increased shift costs by $186 \mathrm{msec}$ in the latter [900-100] group, as compared with only $20 \mathrm{msec}$ in the 100-900 group.) In fact, when we analyzed the performance of the 100-900 group separately, although the influence of mapping reversal was still present in RTs $(85 \mathrm{msec})$ on both switch and repeat trials $\left[F(1,15)=12.38, M S_{\mathrm{e}}=18,877\right]$, it no longer affected shift costs $(F<1)$. This shows that cue-based task preparation counteracted the effect of stimulus-based priming on shift costs, even though the priming effect was still clearly present in overall RTs. 
The analysis of error rates showed results that are generally in line with the RT results (see Table 1). We will summarize the effects only briefly. The ANOVA revealed significant main effects of mapping reversal $[F(1,30)=17.64$, $M S_{\mathrm{e}}=0.002945$; note that the $M S_{\mathrm{e}}$ s for error rates refer to error proportions, whereas Table 1 shows error percentages], task transition $\left[F(1,30)=9.26, M S_{\mathrm{e}}=0.003391\right]$, and congruence $\left[F(1,30)=54.49, M S_{\mathrm{e}}=0.005181\right]$. The increase in error rates due to mapping reversal was slightly but not significantly larger for the 900-100 group than for the 100-900 group $\left[F(1,30)=3.21, M S_{\mathrm{e}}=0.002945, p=\right.$ .083]. Unlike the RT data, the interaction of task transition and response congruence was significant in the errors $\left[F(1,30)=5.79, M S_{\mathrm{e}}=0.003803\right]$, indicating that shift costs were higher for response-incongruent than for response-congruent stimuli (we observed a similar result in the decay contrast, reported below). Also, confirming the numerical trend in the RT data, response congruence interacted with mapping reversal $\left[F(1,30)=16.40, M S_{\mathrm{e}}=\right.$ $0.002803]$, showing that the increase in error rates with mapping reversal was larger for response-incongruent than for response-congruent stimuli. This suggests that some direct S-R priming occurred, over and above the stimulusbased priming of the previously associated task. No other effects were significant (all $p \mathrm{~s}>.11$ ).

In sum, with a short preparation interval (the 900-100 group) the effect of stimulus-based priming was larger for switch than for repeat trials, resulting in markedly increased shift costs after the mapping reversal. In contrast, after a long task-cuing interval (the 100-900 group), the effect of stimulus-based priming on shift costs was practically eliminated, even though substantial priming effects remained in overall RTs. This finding is particularly striking, given that we used a between-subjects manipulation of preparation time. As compared with within-subjects manipulations, the present between-subjects manipulation might have led to less clear-cut preparation-related reductions in shift cost (Altmann, 2004; Koch, 2001, 2005). In fact, the interaction of group and task transition was not significant for either RT $(F<1)$ or error rates $[F(1,30)=$ 2.66, $\left.M S_{\mathrm{e}}=0.003391, p>.10\right]$; we nevertheless found a clear preparation effect with respect to stimulus-based priming, thus validating the present manipulation. We thus conclude that cue-based preparation can counteract involuntary, stimulus-based task priming.

The pattern of between-group effects also speaks against a possible alternative interpretation of our stimulus-based priming effects - an interpretation in terms of priming from the task cues to the stimuli previously associated with them, rather than priming from stimuli to tasks. Our own interpretation is that the previously learned stimulustask associations resulted in bottom-up activation from the stimuli to the task; however, one might argue, instead, that a similar learned association could result in the task cue priming the set of stimuli previously associated with that cue. Mapping reversal would thus have the effect that task cues are now followed by unexpected stimuli. However, this alternative account could not explain why a long CSI should reduce the effect of mapping reversal. On the contrary, this interpretation should predict the opposite-that is, that a longer cuing time would, if anything, increase the priming of the cue-associated stimuli, thus producing an even larger cost of stimulus-task reversal, rather than a reduced effect, as was observed. Therefore, we maintain our assumption that the stimulus-task associations, which we manipulated in pre- and postreversal, resulted in stimulusbased priming of the previously associated task.

\section{Stimulus-Based Priming and Decay of Task Activation From the Preceding Trial}

To test whether stimulus-based priming interacted with possible decay of activation from the preceding trial as a function of increases in RCI, we compared performance between the 900-100 and the 100-100 groups (see the center and right graphs of Figure 2). We carried out an ANOVA on RT in Blocks 4 and 5, with the within-subjects variables of mapping reversal (Block 4 vs. 5), task transition (switch vs. repetition), and response congruence and the between-subjects variable of RCI (900-100 vs. 100-100 group). To avoid redundancy, we will focus on significant effects that include the group variable and will report the other effects only briefly.

Table 1

Mean Error Rates (in Percentages;

With Standard Deviations) as a Function of

Task Transition, Congruence, Mapping Reversal (Prereversal

Block 4 vs. Postreversal Block 5), and Experimental Group

\begin{tabular}{|c|c|c|c|c|c|c|c|c|}
\hline \multirow[b]{3}{*}{ Condition } & \multicolumn{4}{|c|}{ Prereversal } & \multicolumn{4}{|c|}{ Postreversal } \\
\hline & \multicolumn{2}{|c|}{ Switch } & \multicolumn{2}{|c|}{ Repeat } & \multicolumn{2}{|c|}{ Switch } & \multicolumn{2}{|c|}{ Repeat } \\
\hline & $M$ & $S D$ & $M$ & $S D$ & $M$ & $S D$ & $M$ & $S D$ \\
\hline \multicolumn{9}{|l|}{$100-900$ group } \\
\hline Congruent & 3.1 & 3.9 & 2.7 & 3.5 & 2.6 & 4.5 & 2.2 & 4.4 \\
\hline Incongruent & 7.7 & 5.8 & 7.6 & 7.8 & 13.1 & 7.8 & 9.9 & 6.0 \\
\hline \multicolumn{9}{|l|}{$900-100$ group } \\
\hline Congruent & 2.6 & 3.8 & 2.6 & 4.9 & 3.8 & 6.7 & 3.1 & 4.8 \\
\hline Incongruent & 8.3 & 8.2 & 3.2 & 5.0 & 16.9 & 13.1 & 9.1 & 8.0 \\
\hline \multicolumn{9}{|l|}{$100-100$ group } \\
\hline Congruent & 2.4 & 4.4 & 3.5 & 4.5 & 3.7 & 4.4 & 2.5 & 5.2 \\
\hline Incongruent & 10.3 & 9.2 & 5.4 & 5.7 & 15.6 & 12.3 & 7.3 & 5.5 \\
\hline
\end{tabular}


The ANOVA revealed significant main effects of mapping reversal $\left[F(1,30)=87.270, M S_{\mathrm{e}}=37,696\right]$, task transition $\left[F(1,30)=167.74, M S_{\mathrm{e}}=18,404\right]$, and response congruence $\left[F(1,30)=55.42, M S_{\mathrm{e}}=3,430\right]$ and significant two-way interactions of mapping reversal and task transition $\left[F(1,30)=42.25, M S_{\mathrm{e}}=12,634\right]$ and task transition and response congruence $[F(1,30)=7.21$, $\left.M S_{\mathrm{e}}=5,978\right]$. The interaction of mapping reversal and response congruence was also significant $[F(1,30)=4.81$, $\left.M S_{\mathrm{e}}=6,395\right]$, suggesting that the effect of mapping reversal was somewhat greater for response-incongruent than for response-congruent stimuli. (Note that the equivalent interaction for the preparation contrast, reported above, was not significant in the RT data, but only in the error rates.) This two-way interaction appears to be due mainly to the larger response congruence effects in the 100-100 group; however, more critically, the relevant three-way interaction of mapping reversal, congruence, and group was not, in fact, significant $\left[F(1,30)=1.38, M S_{\mathrm{e}}=6,395\right.$, $p>.2]$.

In the present context, the theoretically important effects are those that include the group variable. The main effect of group was significant $\left[F(1,30)=12.01, M S_{\mathrm{e}}=95,542\right]$, indicating that RTs were generally longer with a short than with a long decay time $(M=901 \mathrm{msec}, S E=22 \mathrm{msec}$, vs. $M=767 \mathrm{msec}, S E=32 \mathrm{msec}$ ). More specifically we expected that a short decay time should increase RT on switch trials, because residual activation of the competing, incorrect task should be greater than after a long decay time, and this was the case (190-msec group difference in mean RT). However, a short RCI also increased RTs on task repetition trials [by $78 \mathrm{msec} ; F(1,30)=6.59, M S_{\mathrm{e}}=$ $29,436]$. This effect was not expected, because in case of a task repetition, residual activation from the preceding trial should be beneficial, rather than interfering. This is a potentially interesting result that needs further clarification. It is also possible that, for some unknown reason, the participants in the 100-100 group were generally slower than those in the 900-100 group. In that case, the predicted interaction of the group variable with task transition would be the relevant finding, and this interaction was indeed significant $\left[F(1,30)=10.93, M S_{\mathrm{e}}=18,404\right.$, $p<.002$ ], showing that, as was predicted, shift costs were larger after a short than after a long decay time (276 vs. $164 \mathrm{msec})$. This interaction was further modulated by the response congruence variable $\left[F(1,30)=11.76, M S_{\mathrm{e}}=\right.$ $5,978, p=.002]$; that is, the increase in shift costs with the shorter decay interval was even greater on responseincongruent than on congruent trials.

In contrast, there was no significant two-way interaction between mapping reversal and group $(F=0.21, p>$ .64). This shows that, contrary to our original expectation, the manipulation of decay time, although it affected shift costs, did not significantly influence stimulus-based priming (see below). No other RT effects were significant $(p s>$.14). Error rates were examined for the possibility of a speed-accuracy trade-off. There were no significant effects involving the group variable, and there was no in- dication of a trade-off. Therefore, the analyses will not be reported in detail.

In sum, we found that decay time affected shift costs, suggesting that shift costs include an important but rapidly dissipating component that is due to carryover of activation of the preceding task set, or task set inertia (see, e.g., Allport et al., 1994; Allport \& Wylie, 1999; Altmann, 2002; Sohn \& Anderson, 2001). However, decay time did not influence the effects of stimulus-based priming. We will return to this observed independence of decay and stimulus-based task priming in the Discussion section.

\section{DISCUSSION}

\section{Stimulus-Based Priming of Tasks}

The present study was conducted in order to explore the effects of stimulus-based priming on task switching. We manipulated stimulus-based priming by establishing unique associations between individual stimulus items and tasks. After several hundreds of trials of practice with this fixed stimulus-to-task mapping, we reversed the mapping. RT greatly increased with the mapping reversal. This showed that stimulus-based priming had a substantial effect. This result confirms and extends earlier demonstrations of stimulus-based priming, in which switching between Stroop tasks was studied (Allport \& Wylie, 2000; Waszak et al., 2003, 2004, 2005). In the present study, we used a cuing paradigm with a pair of numerical judgment tasks. Importantly, our priming procedure was identical for both tasks, whereas the earlier studies had focused on the priming effect in one direction only (i.e., on the wordreading task). On the basis of the present results, we may conclude that stimulus-based priming in task switching is a general phenomenon.

In addition to extending the generality of stimulus-based priming effects in task switching, we addressed the question of whether stimulus-based priming is based on prior learning of stimulus-task associations or of stimulus-response (S-R) associations. To answer this question, we used both response-incongruent stimuli (like Allport \& Wylie, 2000; Waszak et al., 2003) and response-congruent stimuli. For response-congruent stimuli, previously learned S-R associations obviously could not generate interference, because for these stimuli the $\mathrm{S}-\mathrm{R}$ associations remained unchanged; mapping reversal changed only the tasks (including the relevant judgment categories) with which these stimuli were associated. Finding priming effects for response-congruent stimuli would thus imply priming at the task level. In fact, we found strong priming effects for response-congruent, as well as for response-incongruent, stimuli, thus clearly demonstrating stimulus-based priming of tasks.

A possible objection to our interpretation of the results might be that participants learn to reframe the experimental situation as comprising eight different, fixed S-R associations, instead of using two different, competing categorization rules (see Logan \& Bundesen, 2003). However, the data argue against this hypothesis. First, even in 
Block 4, there were clear response congruence effects on switch trials. This indicates the influence of stimulus categorization, because learning of fixed S-R associations could not explain why there should be increased competition with incongruent stimuli. Second, as was mentioned above, the fact that RT for response-congruent stimuli also increased with mapping reversal shows even more strongly that the acquisition of simple S-R associations could not explain the data pattern, since for these stimuli the $\mathrm{S}-\mathrm{R}$ associations remained unchanged.

Having established that the imperative stimuli themselves constitute a potent source of task activation-in particular, when the same stimuli occur in the context of different tasks, as in many task-switching paradigms-we now will turn to the question of how this type of stimulusbased priming interacts (1) with cue-based task activation (preparation) and (2) with the dissipation of task activation from a previous trial (decay).

\section{Stimulus-Based Priming and Cue-Based Preparation of Tasks}

We found that with a long cuing interval, cue-based task preparation markedly reduced the stimulus-based priming effect on task repetition trials, but even more strongly on task switch trials. To account for this effect, we assume that cue-based preparation of the correct task dampens the effect of stimulus-based priming of the competing task. That is, if task competition on a task switch trial is already substantially resolved as a result of cue-based activation of the correct task after a long CSI, stimulus-based priming of the competing task should be expected to have little impact, as indeed we found. Gilbert and Shallice (2002) recently proposed a connectionist model that is able to model effects of stimulus-based task priming by associative learning, but the authors did not attempt to model the influence of preparation on this priming effect. We believe that our finding represents an observation to which formal models of task switching should be addressed. It would be interesting to know how the Gilbert and Shallice model might be adapted to accommodate the observed reduction of stimulus-based priming following a long CSI.

Our finding that cue-based task preparation substantially reduced the effect of stimulus-based priming on RT shift costs is also theoretically important, because stimulus-based priming might have been a candidate as an account for the residual shift costs that persist even after long preparation intervals (see, e.g., Allport \& Wylie, 2000; De Jong, 2000; Hübner, Futterer, \& Steinhauser, 2001; Meiran, 1996; Nieuwenhuis \& Monsell, 2002; Rogers \& Monsell, 1995). The fact that the RT contribution of stimulus-based priming to shift costs was largely eliminated after a long CSI suggests that stimulus-based priming is probably not a sufficient explanation for residual shift costs. We suggest that residual shift costs are due primarily to the involuntary persistence of activation from the preceding task (see Allport et al., 1994), which, it appears, cannot be entirely overcome by cue-based (i.e., intentional) task preparation (see also Rogers \& Monsell, 1995; Rubinstein, Meyer, \& Evans, 2001; Sohn \& Ander- son, 2001). The explanation for the latter finding will be discussed below and in the next section.

An important proposal put forward by Meiran (2000b) is that task set can be decomposed into a stimulus set component (e.g., a bias in favor of the task-relevant stimulus dimension[s] and higher order stimulus categories) and a response set component (e.g., the association of stimulus category and response). Both these components, we assume, persist involuntarily into the following trial, thus facilitating a task repetition and interfering with a task switch, to produce carryover shift costs (Koch \& Philipp, 2005; Schuch \& Koch, 2003, 2004). For a pair of tasks with overlapping (bivalent) responses, Meiran (2000b) found that shift costs were not eliminated by cue-based preparation, even after a long CSI; in contrast, for tasks with nonoverlapping (univalent) responses, shift costs disappeared completely after a long CSI. This suggests that the component of task set that persists (and hence, contributes to shift costs) even after a long CSI is related to the between-task interference due to overlapping responses (i.e., to response set). In contrast, the stimulus set component of task set is strongly affected by CSI. Cuebased preparation, in other words, affects stimulus set but has comparatively little effect on response set (see also Meiran, 2000a; Schuch \& Koch, 2003).

If the argument above is correct-namely, that cuebased preparation affects primarily stimulus set, rather than response set-this has important implications also for the understanding of stimulus-based priming. Cuebased preparation in our experiment (after a long CSI) eliminated the contribution of stimulus-based priming to shift costs. Accordingly, we speculate that stimulus-based priming, like cue-based preparation, relates primarily to stimulus set. That is, we suggest that the stimulus reactivates its associated stimulus category (e.g., odd). Conceivably, this activation spreads also to the other member of the task-relevant set of stimulus categories (e.g., oddeven), but further research will have to test this.

Our observation that task preparation greatly reduced the impact of stimulus-based task priming appears to be conceptually similar to the finding recently reported by Yeung and Monsell (2003a) that preparation can reduce task-priming effects that are based on practice-related manipulations of task strength. This converging evidence for a biasing role of preparation with respect to the effects of task priming is encouraging with respect to the issue of understanding the functional mechanism underlying preparation effects.

In view of the present findings, we suggest that task preparation may be understood as an associative cue-totask activation process, biasing the system in favor of task-relevant versus task-irrelevant elements (see Meiran, 2000b). This suggestion would be consistent with models of attention and executive processes in the form of integrated competition networks (e.g., Duncan, 1996; Duncan et al., 1997; Miller \& Cohen, 2001) or biased competition (see Miller, 2000; Miller \& Cohen, 2001). That is, on the basis of associative learning, environmental cues (both instructed task cues and the stimuli themselves) and inter- 
nal cues (memories, intentions) become associated with states of activation of task-relevant neural components in competing brain systems (or modules). On the basis of this learning history, task cues can come to exert an indirect bias on the competition of different pathways. In this way, task competition can be resolved, but at the cost of additional time for the system to settle into a stable state (see, e.g., Gilbert \& Shallice, 2002; Ward, 1999).

The preceding discussion raises the question as to whether cue-based (and thus, by definition, voluntary) biasing of task competition might be a theoretical alternative to the well-known reconfiguration view (e.g., Monsell, 2003). We believe that our view that task performance is governed by biased competition is not mutually exclusive with the reconfiguration view. Rather, we believe that reconfiguration is an essentially figurative term, which actually refers to functional mechanisms very much like those referred to above. If so, the only critical issue between these two approaches is whether one assumes that there is a unique switching process that comes into play only on task switch trials and does not occur on task repetitions (e.g., Rogers \& Monsell, 1995; Rubinstein et al., 2001). More recently, it appears, the latter assumption has come to be viewed increasingly skeptically (e.g., Gilbert \& Shallice, 2002; Koch, 2005; Mayr \& Kliegl, 2003; Yeung \& Monsell, 2003b). For example, in Gilbert and Shallice's model, it is assumed that "precisely the same computational processes occur on switch and nonswitch trials. The only difference between the two types of trials is in the initial state of the network" (p. 331). At any rate, we believe that research within the framework of biased task competition, affected by priming (or biasing) from multiple sources of activation, may eventually help to formulate a unifying account of task performance.

\section{Stimulus-Based Priming and Decay}

Our results referring to decay time effects support the idea of gradually decaying activation from the preceding task. We found that a longer RSI resulted not only in a shorter RT generally, but also in smaller shift costs. However, although a longer decay interval since the preceding trial reduced shift costs (see Koch, 2001; Meiran et al., 2000), it reduced neither the main effect of stimulus-based priming nor its interaction with task transition (i.e., its effect on shift costs).

To account for this apparent independence of the effects of decay time and of stimulus-based priming, we offer the following speculation. In the preceding section, we argued that both stimulus-based priming and cue-based preparation relate primarily to stimulus-related components of task set (stimulus set; Meiran, 2000b), which would explain why these two variables interact. We now extend this argument to speculate that decay time (i.e., RSI) effects relate, on the contrary, to response set. If so, given that RSI and stimulus-based priming relate to different task set components, their effects should indeed be independent of each other.

Indirect support for this proposal comes from Schuch and Koch (2003) and Koch, Gade, and Philipp (2004), who argued that $n-2$ task repetition costs (backward inhibition effects) can also be related to response set. In accord with our present proposal, it is noteworthy that previous studies have shown no effect of varying the CSI on backward inhibition (e.g., Mayr \& Keele, 2000; Schuch \& Koch, 2003). Our suggestion is that it is the response-related component of task set that decays, comparatively rapidly, after response execution (i.e., as a function of RSI), whereas the stimulus-related component may persist with relatively little passive change over this time scale, unless or until it is counteracted by the cue-based preparation of a different task. (We assume, however, that the response set component does not decay completely. In contrast, a robust bias remains, unaffected by cue-based preparation, resulting in residual shift costs, even after a long CSI.) Shift costs in general should be affected both by cue-based preparation (CSI) and by RSI (as indeed was observed), because shift costs, as was discussed earlier, are due to persisting task biases that include both stimulus-related and response-related components.

These speculative proposals are open to empirical test, and it will be interesting to examine the dynamics of the postresponse decay of task activation in more detail. Clearly, at this point, we admit that our explanation for the observed independence of stimulus-based priming and RSI is speculative, but we believe that it holds considerable theoretical promise.

On a more general theoretical level, the notion of decay of task activation seems to fit quite naturally into the present activation-based framework. However, given that the assumption of decay processes is rather fundamental to activation-based models (Altmann, 2002; Gilbert \& Shallice, 2002; Sohn \& Anderson, 2001; Yeung \& Monsell, 2003b), it is surprising that there is comparatively little empirical research on decay time effects in task switching. We hope that the present data on decay time effects may prove useful for modelers who wish to specify further the nature and time course of decay processes.

\section{Open Issues}

With respect to a more general account of task performance, we note that, thus far, we have neglected the issue of whether stimulus-based task priming is facilitatory only or whether it can also be inhibitory. Generally, we have assumed that the major construct that explains task performance in task switching is task activation (as in the model proposed by Yeung \& Monsell, 2003b). However, experiments on switching among three different tasks have provided increasing evidence for inhibitory processes also (see, e.g., Gade \& Koch, 2005; Koch et al., 2004; Mayr \& Keele, 2000; Philipp \& Koch, in press; Schuch \& Koch, 2003), and Waszak et al. (2005) have identified conditions in which stimulus-based priming can include a true negative-priming component. Further theoretical effort will be required to integrate inhibitory processes into the current activation-based account.

Another open issue concerns the origin of task mixing costs (i.e., the phenomenon that RT for task repetitions in a task-switching block is typically higher than that in 
task-pure blocks; see Los, 1996). In our data, even after a long task-cuing interval, stimulus-based priming still had a considerable effect. However, as has already been noted, the priming effect was then equally strong on switch and repeat trials, suggesting that stimulus-based priming may not account for residual switch costs. We speculate that this persisting stimulus-based priming effect may be one of the causes of task mixing costs. Recent evidence (with a completely different pair of tasks) supports this speculation (Koch, Prinz, \& Allport, 2005).

\section{Summary and Conclusions}

We observed that cue-based preparation of the correct task and stimulus-based priming of the incorrect task interacted, so that the latter ceased to exert its influence on shift costs when the correct task was already strongly activated (after a long CSI). Increasing the time interval (RSI) since the preceding trial-thus allowing task activation from the previous trial to partially decay-substantially reduced shift costs, in accord with the idea that an (evidently substantial) component of shift costs is due to carryover of task activation (see Allport et al., 1994; Allport \& Wylie, 1999; Sohn \& Anderson, 2001). However, decay time did not influence the stimulus-based priming effect. To account for this complex pattern of results, we assume that shift costs can be decomposed into separate components, related to stimulus set and response set (see Meiran, 2000b). We speculate that CSI and stimulus-based priming affect stimulus set, whereas decay time primarily affects response set. Further research will have to reveal whether these speculations are correct. We are certain, however, that examination of the interactions between these three different sources of task activation, in the context of task switching, is very fruitful theoretically.

\section{REFERENCES}

Allport, D. A., Styles, E. A., \& Hsieh, S. (1994). Shifting intentional set: Exploring the dynamic control of tasks. In C. Umiltà \& M. Moscovitch (Eds.), Attention and performance XV: Conscious and nonconscious information processing (pp. 421-452). Cambridge, MA: MIT Press, Bradford Books.

Allport, [D.] A., \& Wylie, G. (1999). Task-switching: Positive and negative priming of task-set. In G. W. Humphreys, J. Duncan, \& A. Treisman (Eds.), Attention, space, and action: Studies in cognitive neuroscience (pp. 273-296). Oxford: Oxford University Press.

Allport, [D.] A., \& Wylie, G. (2000). "Task-switching," stimulusresponse bindings, and negative priming. In S. Monsell \& J. Driver (Eds.), Control of cognitive processes: Attention and performance XVIII (pp. 35-70). Cambridge, MA: MIT Press.

Altmann, E. M. (2002). Functional decay of memory for tasks. Psychological Research, 66, 287-297.

Altmann, E. M. (2004). The preparation effect in task switching: Carryover of SOA. Memory \& Cognition, 32, 153-163.

BRAVER, T. S., \& COHEN, J. D. (2000). On the control of control: The role of dopamine in regulating prefrontal function and working memory. In S. Monsell \& J. Driver (Eds.), Control of cognitive processes: Attention and performance XVIII (pp. 713-737). Cambridge, MA: MIT Press.

Braver, T. S., Reynolds, J. R., \& Donaldson, D. I. (2003). Neural mechanisms of transient and sustained cognitive control during task switching. Neuron, 39, 713-726.

De Jong, R. (2000). An intention-activation account of residual switch costs. In S. Monsell \& J. Driver (Eds.), Control of cognitive processes: Attention and performance XVIII (pp. 357-376). Cambridge, MA: MIT Press.

Duncan, J. (1996). Cooperating brain systems in selective perception and action. In T. Inui \& J. L. McClelland (Eds.), Attention and performance XVI: Information integration in perception and communication (pp. 549-578). Cambridge, MA: MIT Press, Bradford Books.

DunCAN, J., Humphreys, G., \& WARD, R. (1997). Competitive brain activity in visual attention. Current Opinion in Neurobiology, 7, 255261.

GADE, M., \& КосH, I. (2005). Linking inhibition to activation in the control of task sequences. Psychonomic Bulletin \& Review, 12, 530-534.

Gilbert, S., \& Shallice, T. (2002). Task switching: A PDP model. Cognitive Psychology, 44, 297-337.

GoschKe, T. (2000). Intentional reconfiguration and involuntary persistence in task-set switching. In S. Monsell \& J. Driver (Eds.), Control of cognitive processes: Attention and performance XVIII (pp. 333355). Cambridge, MA: MIT Press.

Hübner, R., Futterer, T., \& Steinhauser, M. (2001). On attentional control as source of residual shift costs: Evidence from twocomponent task shifts. Journal of Experimental Psychology: Learning, Memory, \& Cognition, 27, 640-653.

KocH, I. (2001). Automatic and intentional activation of task sets. Journal of Experimental Psychology: Learning, Memory, \& Cognition, 27, 1474-1486.

KocH, I. (2005). Sequential task predictability in task switching. Psychonomic Bulletin \& Review, 12, 107-112.

Koch, I., Gade, M., \& PhilipP, A. (2004). Inhibition of response mode in task switching. Experimental Psychology, 51, 51-57.

Koch, I., \& Philipp, A. (2005). Effects of response selection on the task repetition benefit in task switching. Memory \& Cognition, 33, 624-634.

Koch, I., Prinz, W., \& Allport, A. (2005). Involuntary retrieval in alphabet-arithmetic tasks: Task-mixing and task-switching costs. Psychological Research, 69, 252-261.

Logan, G. D., \& Bundesen, C. (2003). Clever homunculus: Is there an endogenous act of control in the explicit task cuing procedure? Journal of Experimental Psychology: Human Perception \& Performance, 29, 575-599.

Logan, G. D., \& Bundesen, C. (2004). Very clever homunculus: Compound stimulus strategies for the explicit task-cuing procedure. Psychonomic Bulletin \& Review, 11, 832-840.

Los, S. A. (1996). On the origin of mixing costs: Exploring information processing in pure and mixed blocks of trials. Acta Psychologica, 94, $145-188$.

Mayr, U., \& Keele, S. W. (2000). Changing internal constraints on action: The role of backward inhibition. Journal of Experimental Psychology: General, 129, 4-26.

MaYr, U., \& KliEgL, R. (2003). Differential effects of cue changes and task changes on task-set selection costs. Journal of Experimental Psychology: Learning, Memory, \& Cognition, 29, 362-372.

Meiran, N. (1996). Reconfiguration of processing mode prior to task performance. Journal of Experimental Psychology: Learning, Memory, \& Cognition, 22, 1423-1442.

Meiran, N. (2000a). Modeling cognitive control in task-switching. Psychological Research, 63, 234-249.

Meiran, N. (2000b). The reconfiguration of stimulus task sets and response task sets during task switching. In S. Monsell \& J. Driver (Eds.), Control of cognitive processes: Attention and performance XVIII (pp. 377-400). Cambridge, MA: MIT Press.

Meiran, N., Chorev, Z., \& SAPIR, A. (2000). Component processes in task switching. Cognitive Psychology, 41, 211-253.

MilleR, E. K. (2000). The prefrontal cortex and cognitive control. Nature Reviews Neuroscience, 1, 59-65.

Miller, E. K., \& CoHEN, J. D. (2001). An integrative theory of prefrontal cortex function. Annual Review of Neuroscience, 24, 167-202.

Monsell, S. (2003). Task switching. Trends in Cognitive Sciences, 7 , 134-140.

Nieuwenhuis, S., \& Monsell, S. (2002). Residual costs in task switching: Testing the failure-to-engage hypothesis. Psychonomic Bulletin \& Review, 9, 86-92. 
PhilipP, A., \& KoCH, I. (in press). The relation of task inhibition and task repetition in task switching. European Journal of Cognitive Psychology.

Rogers, R. D., \& Monsell, S. (1995). Costs of a predictable switch between simple cognitive tasks. Journal of Experimental Psychology: General, 124, 207-231.

Rubinstein, J., Meyer, D. E., \& Evans, J. E. (2001). Executive control of cognitive processes in task switching. Journal of Experimental Psychology: Human Perception \& Performance, 27, 763-797.

ScHuch, S., \& Koch, I. (2003). The role of response selection for inhibition of task sets in task shifting. Journal of Experimental Psychology: Human Perception \& Performance, 29, 92-105.

$\mathrm{SCHUCH}, \mathrm{S}$., \& KoCH, I. (2004). The costs of changing the representation of action: Response repetition and response-response compatibility in dual tasks. Journal of Experimental Psychology: Human Perception \& Performance, 30, 566-582.

Shiffrin, R. M., \& Schneider, W. (1977). Controlled and automatic human information processing: II. Perceptual learning, automatic attending and a general theory. Psychological Review, 84, 127-190.

SoHn, M.-H., \& ANDERSON, J. R. (2001). Task preparation and task repetition: Two-component model of task switching. Journal of Experimental Psychology: General, 130, 764-778.

VAN DER HeIJDEN, A. H. C. (1981). Short-term visual information forgetting. London: Routledge \& Kegan Paul.

WARD, R. (1999). Interactions between perception and action systems: A model for selective attention. In G. W. Humphreys, J. Duncan, \& A. Treisman (Eds.), Attention, space, and action: Studies in cognitive neuroscience (pp. 311-332). Oxford: Oxford University Press.

WaszaK, F., Hommel, B., \& Allport, A. (2003). Task-switching and long-term priming: Role of episodic S-R bindings in task-shift costs. Cognitive Psychology, 46, 361-413.
WaszaK, F., Hommel, B., \& Allport, A. (2004). Semantic generalization of stimulus-task bindings. Psychonomic Bulletin \& Review, 11, 1027-1033.

Waszak, F., Hommel, B., \& Allport, A. (2005). Interaction of task readiness and automatic retrieval in task switching: Negative priming and competitor priming. Memory \& Cognition, 33, 595-610.

Yeung, N., \& Monsell, S. (2003a). The effects of recent practice on task switching. Journal of Experimental Psychology: Human Perception \& Performance, 29, 919-936.

Yeung, N., \& Monsell, S. (2003b). Switching between tasks of unequal familiarity: The role of stimulus-attribute and response-set selection. Journal of Experimental Psychology: Human Perception \& Performance, 29, 455-469.

\section{NOTES}

1. In fact, the participants performed a further three blocks with reversed mapping; however, we focus on the immediate effect of reversalinduced, stimulus-based priming when it was at its strongest- that is, in Block 5-as a function of cue-based task preparation and decay time.

2. Note that preparation time is more commonly varied within subjects. However, in the context of the present experimental design, we had only 24 observations for each of the four combinations of task transition and response congruence in Blocks 4 and 5, respectively. Therefore, in order not to further reduce the number of observations in each cell of the design, we used a between-subjects variation of CSI.

(Manuscript received December 22, 2003; revision accepted for publication March 18, 2005.) 